\title{
Carbon tips as electrodes for single-molecule junctions
}

\author{
Andres Castellanos-Gomez, ${ }^{1, a), b)}$ Stefan Bilan, ${ }^{2}$ Linda A. Zotti, ${ }^{2}$ Carlos R. Arroyo, ${ }^{1, b)}$ \\ Nicolás Agraït, ${ }^{1,3,4}$ Juan Carlos Cuevas, ${ }^{2, a)}$ and Gabino Rubio-Bollinger ${ }^{1,3, a)}$ \\ ${ }^{1}$ Departamento de Física de la Materia Condensada (C-III), Universidad Autónoma de Madrid, \\ Campus de Cantoblanco, 28049 Madrid, Spain \\ ${ }^{2}$ Departamento de Física Teórica de la Materia Condensada (C-III), Universidad Autónoma de Madrid, \\ Campus de Cantoblanco, 28049 Madrid, Spain \\ 3 Instituto Universitario de Ciencia de Materiales "Nicolás Cabrera," Campus de Cantoblanco, \\ 28049 Madrid, Spain \\ ${ }^{4}$ Instituto Madrileño de Estudios Avanzados en Nanociencia IMDEA-Nanociencia, 28049 Madrid, Spain
}

(Received 15 July 2011; accepted 27 August 2011; published online 20 September 2011)

\begin{abstract}
We study electron transport through single-molecule junctions formed by an octanethiol molecule bonded with the thiol anchoring group to a gold electrode and the opposing methyl endgroup to a carbon tip. Using the scanning tunneling microscope based break junction technique, we measure the electrical conductance of such molecular junctions. We observe the presence of well-defined conductance plateaus during the stretching of the molecular bridge, which is the signature of the formation of a molecular junction. (c) 2011 American Institute of Physics. [doi:10.1063/1.3643031]
\end{abstract}

Understanding electron transport through a single molecule is the main goal in molecular electronics. A primary aim is to find ways to form a reliable mechanical and electrical connection between the molecule under study and the macroscopic electrodes. The mechanical and electrical properties of a molecular junction can be tuned by a clever design of the molecular structure but also by a proper selection of the material of the electrodes. So far, gold has been the main material of choice for the electrodes in scanning tunneling microscope break junction (STM-break junction) experiments because gold is chemically inert, even in air, while molecules can be bound to it with well characterized linker groups such as thiols ${ }^{1-3}$ or amines. ${ }^{1-3}$ However, the bond formation between gold electrodes and methyl terminated molecules such as alkane(mono)thiols, although possible in certain electrochemical environments, ${ }^{4}$ has proven to be elusive in break-junction experiments. ${ }^{5,6}$ This explains why the use of gold as electrodes in single-molecule electronics has limited the variety of molecular systems that can be studied with STM-break junction experiments. In this sense, it would be highly desirable to widen the list of materials that can be used as electrodes. ${ }^{2,7}$ Carbon is obviously a good candidate as an electrode material in molecular electronics because it offers the possibility to contact a variety of molecules without the need of anchoring groups, it is also relatively inert, and it forms structures like graphene ${ }^{8,9}$ and nanotubes ${ }^{10}$ that can be used as nanoelectrodes.

In this work, we have explored the suitability of carbonbased tips as electrodes to form molecular junctions. Using the STM-break junction technique, we have measured the electrical conductance of several hundred octanethiol-based single-molecule bridges $\left(\mathrm{CH}_{3}-\mathrm{C}_{7} \mathrm{H}_{14}-\mathrm{SH}\right)$ in which the thiol anchoring group is bound to a gold electrode and the methyl

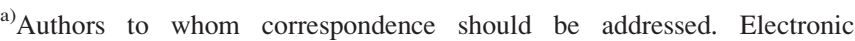
addresses: a.castellanosgomez@tudelft.nl, juancarlos.cuevas@uam.es, and gabino.rubio@uam.es.

b) Present address: Kavli Institute of Nanoscience, Delft University of Technology, Lorentzweg 1, 2628 CJ Delft, The Netherlands.
}

group is linked to a carbon electrode. Most of the conductance traces measured while stretching the molecular junction show a marked plateau which is the signature of the formation of molecular junctions. ${ }^{11,12}$

In order to form single-molecule junctions with a carbon electrode, we used a homebuilt STM (Ref. 13) with a carbon fiber tip. These tips have been characterized for their use in STM proving that the carbon tip apex is not contaminated. ${ }^{14}$ We have also ruled out the possibility that the carbon tip is contaminated or wetted by gold atoms of the surface. ${ }^{15}$

Single-molecule junctions are obtained by repeatedly forming and breaking the contact between the tip and a gold substrate covered with a self-assembled monolayer (SAM) of molecules. We choose octanethiol molecules as a model system because it has the following advantages. First, SAMs of octanethiol molecules can be routinely obtained and are well-characterized by STM studies. ${ }^{16}$ Second, octanethiol is the shortest, and thus most conductive, alkanethiol that forms highly ordered standing-up phase monolayers. ${ }^{17}$ Third, the strong bonding between the thiol group and the gold substrate prevents the tip from pulling the molecules off the substrate during the formation and breaking of the contacts.

We characterized the SAM using STM topography images at constant current with a tunneling resistance of 187 $\mathrm{M} \Omega$. The images in Figure 1 reveal large areas of triangularshaped atomically flat terraces separated by sharp monatomic steps, distinctive of $\mathrm{Au}$ (111) reconstructed surfaces. The higher detail image of Figure 1(b) shows the characteristic stripe-like structures produced by the self-assembly of the alkanethiols into periodic molecular domains. ${ }^{18}$

After the characterization of the SAM surface, we formed single molecule contacts with the carbon tip. We start from a non-contact situation with a tunneling resistance higher than $200 \mathrm{M} \Omega$ at an applied fixed tip bias of $100 \mathrm{mV}$. In order to approach the tip to the sample in a controlled way, the STM current feedback setpoint is lowered to a value of $50 \mathrm{nA}$, corresponding to a resistance of $2 \mathrm{M} \Omega$, in a time lapse of $200 \mathrm{~ms}$. The tip approach is sufficient to bring the carbon tip into gentle contact with the SAM but small 


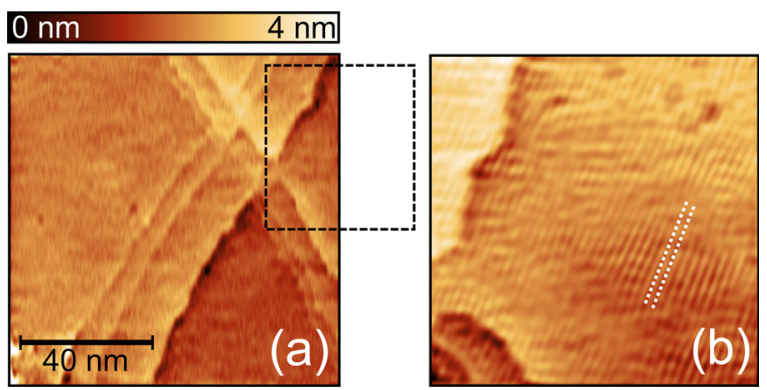

FIG. 1. (Color online) (a) Constant current STM topography image of atomically flat $\mathrm{Au}$ (111) terraces covered by a dense octanethiol monolayer $\left(I_{\text {tunnel }}=1.6 \mathrm{nA}, V_{\text {tip }}=-300 \mathrm{mV}\right)$. (b) Detail of the region marked by the dashed square in (a). The parallel dotted white lines indicate the direction of the characteristic stripe-like structure.

enough not to touch the gold surface. After the shallow contact is established, the current feedback is turned off and the tip is retracted $2 \mathrm{~nm}$ away from the surface at a rate of 60 $\mathrm{nm} / \mathrm{s}$. During this retraction, we measure the current at a fixed bias. A comparison between the STM topographic images before and after the indentation demonstrates that deeper indentation of the tip causes irreversible damage of the SAM. The indentation-retraction cycle is completed by turning the STM feedback loop on. This cycle is repeated 32 times at a predetermined spot of the surface. We have studied 20 different spots of the sample separated by approximately $50 \mathrm{~nm}$.

Several traces of conductance as a function of tip retraction distance $G(z)$ are shown in Figure 2(a). The conductance traces show plateaus at specific values which are the signature of the formation of molecular junctions. ${ }^{17}$ The plateaus are separated by a sharp conductance drops related to the rupture of the molecular bridge. The conductance plateaus do not always occur at the same conductance values because of variations in the microscopic details of the molecular arrangement between the electrodes. A statistical analysis over a large number of molecular junctions is thus necessary. To overcome junction-to-junction fluctuations, we have performed a statistical analysis in which all junction realizations, without sifting conductance traces, are represented as a histogram. ${ }^{13}$ Figure 2(b) shows the conductance histogram (dark tone histogram) with a broad hump centered at $G=8 \times 10^{-6} G_{0}$, where $G_{0}=77.5 \mu \mathrm{S}$ is the conductance quantum, and a smooth and monotonically decreasing background. The hump is associated with the presence of conductance plateaus in individual traces and the background with tunneling conduction. Indeed, the individual traces in Figure 2(a) show that, except at the plateau, the conductance decreases exponentially as a function of the tip separation although its decay constant differs before and after the last conductance plateau. The mean value of the apparent tunneling barrier height before the plateau is $0.6-1.0 \mathrm{eV}$, which can be attributed to tunneling through the SAM. After the molecular bridge breaks, the conduction mechanism is due to vacuum tunneling under ambient conditions ${ }^{19}$ and the mean barrier height is $1.0-2.4 \mathrm{eV}$. The fact that the apparent tunneling barrier height is lower when the tip is in contact with the SAM is in good agreement with previous work in which a lowering of the apparent barrier height is suggested due to the elastic surface deformation induced by the repulsive forces during the tip-sample contact. ${ }^{2}$ Therefore, the background in the conductance histogram can be attributed to vacuum tunneling under ambient conditions for conductance values below the peak (around $G<3 \times 10^{-6} G_{0}$ ) and to tunneling through the SAM for conductance above the peak (around $G>1.5 \times 10^{-5} G_{0}$ ).

We followed the procedure described in Ref. 21 to remove the background tunneling contribution from the histogram in order to better resolve the structure of the conductance hump. Since the slope of the conductance is lower on the plateaus than in the tunneling regions, the tunneling contribution to the conductance histogram can be removed by considering only intervals of the individual $G(z)$ trace whose exponential decay constant is lower than a threshold value. The corrected histogram in Figure 2(b) (light tone histogram) is obtained in this way using a threshold value which corresponds to an apparent barrier height of $450 \mathrm{meV}$. The result is that the background due to the tunneling contribution has been reduced by a factor of 3-4, and the structure of the broad hump in conductance can be better resolved.

We find that the broad hump can be fitted to the sum of two Gaussian peaks in a linear conductance scale whose centers are located at $G_{1}=(5.9 \pm 4.1) \times 10^{-6} \quad G_{0}$ and $G_{2}=(1.3 \pm 0.5) \times 10^{-5} G_{0}$. The presence of multiple peaks in the histogram in previous STM break junction experiments on molecular junctions has been attributed to a

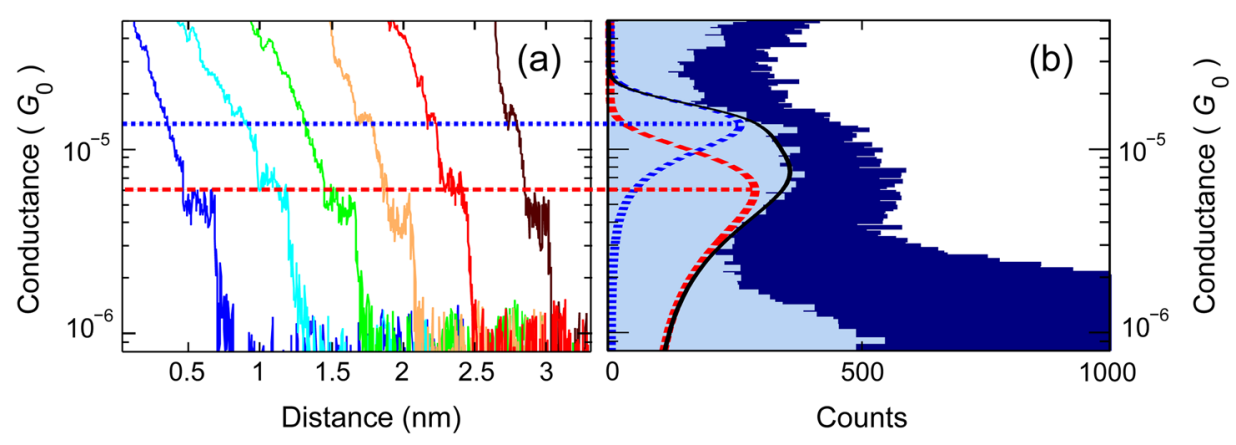

FIG. 2. (Color online) (a) Conductance traces (shifted horizontally by $0.5 \mathrm{~nm}$ for clarity) showing characteristic conductance plateaus. Below a conductance of $1.5 \times 10^{-6} G_{0}$, the measurements are limited by the noise level of the current amplifier. (b) Conductance histogram built from 740 traces (dark tone histogram) and corrected histogram (light tone histogram) after subtracting the tunnelling contribution, following the procedure described in Ref. 20. The corrected histogram shows two conductance peaks. The dashed and the dotted curves are the two Gaussians and the solid black curve is their sum. The maximum of the conductance peaks marks the two most probable molecular configurations (horizontal dashed/dotted lines). 
varying number of molecules contributing to the transport. ${ }^{21}$ The fact that the value of $G_{2}$ is twice that of $G_{1}$ suggests that plateaus at conductance $G_{2}$ correspond to electron transport through two simultaneously connected molecules each of which has a conductance $G_{1}$.

We compare the conductance value of these goldoctanethiol-carbon single molecular junctions with other model systems. The conductance through octanethiols has been studied using gold electrodes in configurations such as 2D nanoparticle arrays ${ }^{13}$ and nanopores ${ }^{22}$ with a relatively large gold-octanethiols-gold junctions (400-1000 molecules are involved). The conductance per molecule has been found to be in the range of $(8-70) \times 10^{-8} G_{0}$ (Ref. 23) which is more than an order of magnitude lower than the value we have obtained for gold-octanethiol-carbon junctions. On the other hand, break junction experiments with octanethiol molecules and gold electrodes show that not only the conductance is low but also is the probability of the formation of molecular junction..$^{22,23}$ The usual approach to provide both good electrical contact and strong chemical bonding to gold electrodes is to functionalize the molecules with thiol anchoring groups on both ends. In single-molecule experiments with octanedithiols, the reported conductance is in the range of $(1-25) \times 10^{-5}$ $G_{0}{ }^{2,7,24}$ The corresponding value for the conductance of the carbon tip contacted octanethiols is comparable in spite of the lack of a specific anchoring group. Thus, the comparison with other model systems supports the suitability of carbon fiber tips to contact methyl terminated molecules.

The appearance of a plateau in the conductance traces suggests the formation of a chemical bond between the alkane chain and the carbon tip. However, it is not easy to say a priori how a methyl-terminated chain can bind to a pure carbon-hydrogen structure. For this reason and in order to shed some light on the formation mechanism of our molecular junctions, we have performed simulations within the framework of density functional theory (DFT) to find/propose a feasible scenario for the formation of a stable alkanecarbon contact. ${ }^{15}$

In conclusion, we have used carbon fiber tips as electrodes in an STM-break junction configuration to form singlemolecule junctions with octanethiol deposited on a gold surface. We find that carbon tips provide a proper mechanical linking to the methyl group allowing the routine formation of single-molecule bridges. The conductance traces during the stretching of the bridges show well defined plateaus that are reflected in the conductance histogram as a prominent peak at $5.9 \times 10^{-6} G_{0}$ which corresponds to the conductance of a single molecule. This value is comparable to that of octanedithiol between gold electrodes. It is important to emphasize that these carbon tips can be suitable candidates to contact a variety of organic molecules and they can also be combined with other substrate materials including carbon itself to form purely organic single-molecule devices.

The authors wish to acknowledge the help of R. H. M. Smit and E. Leary for carefully reading the manuscript and for useful discussions. A.C-G. acknowledges fellowship support from the Comunidad de Madrid (Spain). This work was supported by MICINN (Spain) through the programs MAT2008-01735 and CONSOLIDER-INGENIO-2010 CSD-2007-00010, by Comunidad de Madrid through the program NANOBIOMAGNET S2009/MAT1726, by the EU through the networks BIMORE (MRTN-CT-2006-035859), and by the EC through the network FP7 ITN "FUNMOLS" Project Number 212942.

${ }^{1}$ M. A. Reed, C. Zhou, C. J. Muller, T. P. Burgin, and J. M. Tour, Science 278(5336), 252 (1997).

${ }^{2}$ X. Li, J. He, J. Hihath, B. Xu, S. M. Lindsay, and N. J. Tao, J. Am. Chem. Soc. 128(6), 2135 (2006).

${ }^{3}$ C. Li, I. Pobelov, T. Wandlowski, A. Bagrets, A. Arnold, and F. Evers, J. Am. Chem. Soc. 130(1), 318 (2008).

${ }^{4}$ E. Wierzbinski and K. Slowinski, Langmuir 22(12), 5205 (2006).

${ }^{5}$ F. L. X. Chen, J. H. Z. Hihath, and N. Tao, J. Am. Chem. Soc. 128(49), 15874 (2006).

${ }^{6}$ S. Y. Quek, L. Venkataraman, H. J. Choi, S. G. Louie, M. S. Hybertsen, and J. B. Neaton, Nano Lett. 7(11), 3477 (2007).

${ }^{7}$ S. Wu, M. T. Gonzalez, R. Huber, S. Grunder, M. Mayor, C. Schonenberger, and M. Calame, Nat. Nanotechnol. 3(9), 569 (2008).

${ }^{8}$ M. Kiguchi, O. Tal, S. Wohlthat, F. Pauly, M. Krieger, D. Djukic, J. C. Cuevas, and J. M. van Ruitenbeek, Phys. Rev. Lett. 101(4), 46801 (2008).

${ }^{9}$ R. H. M. Smit, Y. Noat, C. Untiedt, N. D. Lang, M. C. van Hemert, and J. M. van Ruitenbeek, Nature 419(6910), 906 (2002).

${ }^{10}$ H. W. C. Postma, Nano Lett. 10(2), 420 (2010).

${ }^{11}$ X. Guo, A. A. Gorodetsky, J. Hone, J. K. Barton, and C. Nuckolls, Nat. Nanotechnol. 3(3), 163 (2008).

${ }^{12}$ S. Roy, H. Vedala, A. D. Roy, D. Kim, M. Doud, K. Mathee, H. Shin, N. Shimamoto, V. Prasad, and W. Choi, Nano Lett. 8(1), 26 (2008).

${ }^{13}$ B. Xu and N. J. Tao, Science 301(5637), 1221 (2003).

${ }^{14}$ R. H. M. Smit, R. Grande, B. Lasanta, J. J. Riquelme, G. Rubio-Bollinger, and N. Agrait, Rev. Sci. Instrum. 78(11), 113705 (2007).

${ }^{15}$ See supplementary material at http://dx.doi.org/10.1063/1.3643031 for a detailed description of the tip preparation and a control experiment to rule out the wetting of the tip apex by gold atoms of the surface and DFT simulations.

${ }^{16}$ A. Castellanos-Gomez, N. Agrait, and G. Rubio-Bollinger, Nanotechnology 21(14), 145702 (2010).

${ }^{17}$ M. Sharma, M. Komiyama, and J. R. Engstrom, Langmuir 24(18), 9937 (2008).

${ }^{18}$ G. E. Poirier and E. D. Pylant, Science 272(5265), 1145 (1996).

${ }^{19}$ M. T. Gonzalez, S. Wu, R. Huber, S. J. van der Molen, C. Schönenberger, and M. Calame, Nano Lett. 6(10), 2238 (2006).

${ }^{20}$ Y. A. Hong, J. R. Hahn, and H. Kang, J. Chem. Phys. 108, 4367 (1998).

${ }^{21}$ J. L. Xia, I. Diez-Perez, and N. J. Tao, Nano Lett. 8(7), 1960 (2008).

${ }^{22}$ J. Liao, L. Bernard, M. Langer, C. Schönenberger, and M. Calame, Adv. Mater. 18(18), 2444 (2006).

${ }^{23}$ W. Wang, T. Lee, and M. A. Reed, Phys. Rev. B 68(3), 035416 (2003).

${ }^{24}$ W. Haiss, R. J. Nichols, H. van Zalinge, S. J. Higgins, D. Bethell, and D. J. Schiffrin, Phys. Chem. Chem. Phys. 6(17), 4330 (2004). 
Applied Physics Letters is copyrighted by the American Institute of Physics (AIP). Redistribution of journal material is subject to the AIP online journal license and/or AIP copyright. For more information, see http://ojps.aip.org/aplo/aplcr.jsp 